Research Article

\title{
Qualitative Property of Third-Order Nonlinear Neutral Distributed-Delay Generalized Difference Equations
}

\author{
M. Sumathy ${ }^{D}$, P. Venkata Mohan Reddy, and M. Maria Susai Manuel \\ Department of Science and Humanities, R.M.D Engineering College, Kavaraipettai, Chennai 601 206, India \\ Correspondence should be addressed to M. Sumathy; mmariasusai@gmail.com
}

Received 8 June 2021; Accepted 31 July 2021; Published 24 August 2021

Academic Editor: M Syed Ali

Copyright (C) 2021 M. Sumathy et al. This is an open access article distributed under the Creative Commons Attribution License, which permits unrestricted use, distribution, and reproduction in any medium, provided the original work is properly cited.

This paper investigates the qualitative property of third-order nonlinear neutral distributed-delay generalized difference equations. By utilizing Philos-type technique and Riccati transformation, some oscillation criteria are presented to ensure that every solution of this equation oscillates or converges to zero. To illustrate the significance of our main result, we provide a suitable example.

\section{Introduction}

In several areas, such as electrical circuit analysis, finance insurance, dynamic systems, computing, and physical field, third-order difference equations appeared to scrutinize discrete models, naturally occurring in discrete models pertaining physical, biological, and chemical phenomena (see, for example, [1-8]). In many engineering problems, analyzing the existence of oscillatory solutions performs an essential role. Notably, numerous monographs concern with issues of the existence and multiplicity of solutions using different methods, such as critical point theory, topological degree theory, fixed-point index theory, and Lie theory. In recent years, there has been a continual interest in getting sufficient conditions for oscillatory behavior of different classes of third-order difference equations with or without deviating arguments (see [8-23] and the references cited therein).

The third-order nonlinear neutral distributed-delay generalized differential equation is of the form

$$
\Delta_{\ell}\left(a_{1}(k)\left[\Delta_{\ell}\left(a_{2}(k)\left[\Delta_{\ell} z(k)\right]^{\gamma_{1}}\right)\right]^{\gamma_{2}}\right)+\sum_{s=c}^{d} q(k, s) f(x(k+s \ell-\sigma \ell))=0,
$$

where $z(k)=x(k)+\sum_{s=a}^{b} p(k, s) x(k+s \ell-\tau \ell)$ and $\Delta_{\ell}$ is the forward generalized difference operator well defined by $\Delta_{\ell} x(k)=x(k+\ell)-x(k)$,

$\mathbb{N}_{\ell}\left(k_{0}\right)=\left\{k_{0}, k_{0}+\ell, k_{0}+2 \ell, \ldots,\right\}, k_{0} \in[0, \infty), \ell \in(0, \infty)$, and $a, b, c, d \in \mathbb{N}\left(k_{0}\right)$, which subject to the following conditions:

$$
c_{1} \text { : the sequence }\left\{a_{i}(k)\right\} \text { is positive real and }
$$
$\sum_{k=k_{0}}^{\infty}\left(1 / a_{i}^{1 / \gamma_{i}}(k)\right)=\infty$, for $i=1,2$. $c_{2}:\{p(k, s)\}$ and $\{q(k, s)\}$ are nonnegative real sequences along with $0 \leq p(k) \equiv \sum_{s=a}^{b} p(k, s) \leq p<1$.

$c_{3}: \gamma_{1}$ and $\gamma_{2}$ are a quotient of odd positive integers with $\gamma=\gamma_{1} \gamma_{2}$.

$c_{4}$ : the function $f: \mathbb{R} \longrightarrow \mathbb{R}$ is continuous with $\left(f(x) / x^{\gamma}\right) \geq L>0$, where $x \neq 0$ and $L$ is a constant.

$c_{5}: \quad m_{i}(k)=\left[\left(k-k_{i}-j-\ell\right) / \ell\right], \quad \overline{k_{i}}=k_{i}+j, \quad$ and $j=k-k_{0}-\left[\left(k-k_{0}\right) / \ell\right] \ell$. 
By a solution of equation (1), this means a real sequence $\{x(k)\}$, satisfying equation (1) for all $k \in \mathbb{N}_{\ell}\left(k_{0}\right)$ : the solution $\{x(k)\}$ of equation (1) which satisfies $\sup \{|x(k)|: k \geq K\}>0$, for $K \in \mathbb{N}_{\ell}\left(k_{0}\right)$.

Section 2 provides some standard definitions and proves some lemmas necessary for obtaining the main results. Section 3 offers newest oscillation results for equation (1), and finally, in Section 4, we provide a suitable example to determine the major findings.

\section{Preliminaries}

We illustrate a few basic definitions and primary results in this section that will be included in the forthcoming discussions.
Definition 1 (see [16]). If $x(k), k \in[0, \infty)$ is a real or complex valued function and $\ell \in(0, \infty)$, at that point the generalized difference operators $\Delta_{\ell}$ is predefined as

$$
\Delta_{\ell} x(k)=x(k+\ell)-x(k) \equiv y(k)
$$

and then, its inverse is defined by

$$
x(k)=x\left(k_{0}+j\right)+\sum_{r=0}^{m_{0}(k)} y\left(k_{0}+j+r \ell\right), \quad k \in \mathbb{N}_{\ell}(j)
$$

Definition 2 (see [16]). For $\lambda \in \mathbb{N}(1)$, the generalized polynomial factorial is defined by

$$
k_{\ell}^{(\lambda)}=k(k-\ell)(k-2 \ell), \ldots,(k-(\lambda-1) \ell)=\ell^{k} \frac{\Gamma(1+(k / \ell))}{\Gamma((k / \ell)-(\lambda-1))} .
$$

Lemma 1 (see [16]). Let $\ell \in[0, \infty)$. Then, $\Delta_{\ell}\left(n_{\ell}^{(\lambda)}\right)=$ $(\lambda \ell) n_{\ell}^{(\lambda-1)}$.

Lemma 2 (see [16]). Let $u(n)$ and $v(n)$ be real-valued functions. In addition,

$$
\begin{aligned}
\Delta_{\ell}\{u(k) v(k)\} \\
=u(k+\ell) \Delta_{\ell} v(k)+v(k) \Delta_{\ell} u(k) \\
=v(k+\ell) \Delta_{\ell} u(k)+u(k) \Delta_{\ell} v(k) .
\end{aligned}
$$

Lemma 3. Let $x(k)$ be a positive solution of equation (1). Then, function $z(k)$ has any one of the given properties:
$\left(P_{1}\right): \quad z(k)>0, \quad \Delta_{\ell} z(k)>0, \quad \Delta_{\ell}\left(a_{2}(k)\left[\Delta_{\ell} z(k)\right]^{\gamma_{1}}\right)>0$, $\Delta_{\ell}\left(a_{1}(k)\left[\Delta_{\ell}\left(a_{2}(k)\left[\Delta_{\ell} z(k)\right]^{\gamma_{1}}\right)\right]^{\gamma_{2}}\right)<0$

$\left(P_{2}\right): \quad z(k)>0, \quad \Delta_{\ell} z(k)<0, \quad \Delta_{\ell}\left(a_{2}(k)\left[\Delta_{\ell} z(k)\right]^{\gamma_{1}}\right)>0$, $\Delta_{\ell}\left(a_{1}(k)\left[\Delta_{\ell}\left(a_{2}(k)\left[\Delta_{\ell} z(k)\right]^{\gamma_{1}}\right)\right]^{\gamma_{2}}\right)<0$

In the above properties, $k \geq k_{2}$ for sufficiently large $k_{2} \in N_{\ell}\left(k_{0}\right)$.

Proof. Let $\{x(k)\}$ be a positive solution of equation (1) for every $k \geq k_{0}$. By defining $z(k)$, with $z(k) \geq x(k)>0$ for $k \geq k_{1} \in N_{\ell}\left(k_{0}\right)$, and in addition to equation (1), we have

$$
\Delta_{\ell}\left(a_{1}(k)\left[\Delta_{\ell}\left(a_{2}(k)\left[\Delta_{\ell} z(k)\right]^{\gamma_{1}}\right)\right]^{\gamma_{2}}\right)=-\sum_{s=c}^{d} q(k, s) f(x(k+s \ell-\sigma \ell))<0 .
$$

We can know that $a_{1}(k)\left[\Delta_{\ell}\left(a_{2}(k)\left[\Delta_{\ell} z(k)\right]^{\gamma_{1}}\right)\right]^{\gamma_{2}}$ is clearly a decreasing function on $\left[k_{1}, \infty\right)$ with positive or negative finally. Furthermore, we must have to prove that $a_{1}(k)\left[\Delta_{\ell}\left(a_{2}(k)\left[\Delta_{\ell} z(k)\right]^{\gamma_{1}}\right)\right]^{\gamma_{2}}>0$ for $k \geq k_{1} \geq k_{0}$. Otherwise, we have a constant $M_{1}^{\gamma_{2}}>0$ such that

$$
\Delta_{\ell}\left(a_{2}(k)\left[\Delta_{\ell} z(k)\right]^{\gamma_{1}}\right)<-\frac{M_{1}}{a_{1}^{1 / \gamma_{2}}(k)}<0, \quad \text { for } k \geq k_{1} .
$$

Hence, by Definition 1,

$$
\begin{aligned}
& a_{2}(k)\left[\Delta_{\ell} z(k)\right]^{\gamma_{1}} \\
& \quad \leq a_{2}\left(\overline{k_{1}}\right)\left(\Delta_{\ell} z\left(\overline{k_{1}}\right)\right)^{\gamma_{1}}-M_{1} \sum_{r=0}^{m_{1}(k)} \frac{1}{a_{1}^{1 / \gamma_{2}}\left(\overline{k_{1}}+r \ell\right)} .
\end{aligned}
$$

Letting $k \longrightarrow \infty$ and then using condition $\left(c_{1}\right)$, we have $\lim _{n \longrightarrow \infty} a_{2}(k)\left[\Delta_{\ell} z(k)\right]^{\gamma_{1}}=-\infty$. Subsequently, there exists a $k_{2} \geq k_{1}$ also a constant $M_{2}^{\gamma_{1}}>0$ so that

$$
a_{2}(k)\left[\Delta_{\ell} z(k)\right]^{\gamma_{1}}<-M_{2}^{\gamma_{1}}, \quad \text { for } k \geq k_{2} .
$$

Dividing the above inequality by $a_{2}(k)$ and by applying summation from $k_{2}$ to $k-\ell$, we obtain

$$
z(k)<z\left(\overline{k_{2}}\right)-M_{2} \sum_{t=0}^{m_{2}(k)} \frac{1}{a_{2}^{1 / \gamma_{1}}\left(\overline{k_{2}}+t \ell\right)}
$$

Letting $k \longrightarrow \infty$ and using condition $\left(c_{1}\right)$, we have $z(n) \longrightarrow-\infty$. Thus, $z(n)<0$ eventually which is contradictory with $z(n)>0$. Consequently, $\Delta_{\ell}\left(a_{2}(k)\left[\Delta_{\ell} z(k)\right]^{\gamma_{1}}\right)$ is positive, that is, $\left[\Delta_{\ell}\left(a_{2}(k)\left(\Delta_{\ell} z(k)\right]^{\gamma_{1}}\right)\right]^{\gamma_{2}}>0$ holds.

It can be known from $\Delta_{\ell}\left(a_{2}(k)\left[\Delta_{\ell} z(k)\right]^{\gamma_{1}}\right)>0$, that is, a monotonically increasing sign in the interval $\left[k_{2}, \infty\right)$. Therefore, $\Delta_{\ell} z(k)$ is ultimately either positive or negative. We have only property $\left(P_{1}\right)$ or $\left(P_{2}\right)$ for $\{z(k)\}$. Hence, the proof is completed. 
Lemma 4. Let $\{x(k)\}$ be a positive solution of equation (1), and $z(k)$ has $\left(P_{2}\right)$ of Lemma 3. If

$$
\sum_{t_{2}=0}^{\infty} \frac{1}{a_{2}^{1 / \gamma_{1}}\left(\overline{k_{3}}+t_{2} \ell\right)}\left(\sum_{t_{1}=t_{2}}^{m_{2}(k)} \frac{1}{a_{1}^{\left(1 / \gamma_{2}\right)}\left(\overline{k_{2}}+t_{1} \ell\right)}\left(\left(\sum_{t=t_{1}}^{m_{1}(k)} \sum_{s=c}^{d} q\left(\overline{k_{1}}+t \ell, s\right)\right)\right)^{\left(1 / \gamma_{2}\right)}\right)^{\left(1 / \gamma_{1}\right)}=\infty
$$

then $x(k)$ of equation $(1)$ converges to zero when $k \longrightarrow \infty$.

Proof. Let $\{x(k)\}$ be a positive solution of equation (1). Since $z(k)$ satisfies the property $\left(P_{2}\right)$ of Lemma 3 , thereby there exist $\beta \geq 0$ such that

$$
\lim _{k \rightarrow \infty} z(k)=\beta \geq 0
$$

Now, we shall prove that $\beta=0$. Let $\beta>0$; then, we have $\beta+\varepsilon>z(k)>\beta$ for every $\epsilon>0$, and $k$ is enough large. Choosing $0<\varepsilon<((1-p) / p) \beta$, from $z(k)$, we have

$$
\begin{aligned}
x(k) & =z(k)-\sum_{s=a}^{b} p(k, s) x(k+s \ell-\tau \ell) \\
& >\beta-\sum_{s=a}^{b} p(k, s) x(k+s \ell-\tau \ell)>\beta-p(\beta+\varepsilon)=\frac{\beta-p(\beta+\varepsilon)}{\beta+\varepsilon}(\beta+\varepsilon)>M_{3} z(k),
\end{aligned}
$$

where $M_{3}=((\beta-p(\beta+\varepsilon)) /(\beta+\varepsilon))>0$. Thus, from equation (1) and $\left(c_{4}\right)$, we have

$\Delta_{\ell}\left(a_{1}(k)\left[\Delta_{\ell}\left(a_{2}(k)\left[\Delta_{\ell} z(k)\right]^{\gamma_{1}}\right)\right]^{\gamma_{2}}\right)=-\sum_{s=c}^{d} q(k, s) f(x(k+s \ell-\sigma \ell)) \leq-\sum_{s=c}^{d} q(k, s) L x^{\gamma}(k+s \ell-\sigma \ell)$.

Now, using (13), we obtain

$$
\Delta_{\ell}\left(a_{1}(k)\left[\Delta_{\ell}\left(a_{2}(k)\left[\Delta_{\ell} z(k)\right]^{\gamma_{1}}\right)\right]^{\gamma_{2}}\right) \leq-M_{3}^{\gamma} L \sum_{s=c}^{d} q(k, s) z^{\gamma}(k+s \ell-\sigma \ell)
$$

Summing the abovementioned inequality from $k_{1}$ to $k-$ $\ell$ and from Definition 1, we obtain

$$
-a_{1}\left(\overline{k_{1}}\right)\left[\Delta_{\ell}\left(a_{2}\left(\overline{k_{1}}\right)\left[\Delta_{\ell} z\left(\overline{k_{1}}\right)\right]^{\gamma_{1}}\right)\right]^{\gamma_{2}} \leq-M_{3}^{\gamma} L \sum_{t=0}^{m_{1}(k)} \sum_{s=c}^{d} q\left(\overline{k_{1}}+t \ell, s\right) z^{\gamma}\left(\overline{k_{1}}+t \ell+s \ell-\sigma \ell\right) .
$$

The above equation can also be rewritten as

$$
\Delta_{\ell}\left(a_{2}\left(\overline{k_{1}}\right)\left[\Delta_{\ell} z\left(\overline{k_{1}}\right)\right]^{\gamma_{1}}\right) \geq \frac{\left(M_{3} \beta\right)^{\left(\gamma / \gamma_{2}\right)} L^{1 / \gamma_{2}}}{a_{1}^{1 / \gamma_{2}}\left(\overline{k_{1}}\right)}\left(\sum_{t=0}^{m_{1}(k)} \sum_{s=c}^{d} q\left(\overline{k_{1}}+t \ell, s\right)\right)^{\left(1 / \gamma_{2}\right)} .
$$


Summing again from $k_{2} \geq k_{1}$ to $k-\ell$, we obtain

$$
-\Delta_{\ell} z\left(\overline{k_{2}}\right) \geq \frac{\left(M_{3} \beta\right) L^{1 / \gamma}}{a_{2}^{1 / \gamma_{1}}\left(\overline{k_{2}}\right)}\left(\sum_{t_{1}=0}^{m_{2}(k)} \frac{1}{a_{1}^{1 / \gamma_{2}}\left(\overline{k_{2}}+t_{1} \ell\right)}\left(\sum_{t=t_{1}}^{m_{1}(k)} \sum_{s=c}^{d} q\left(\overline{k_{1}}+t \ell, s\right)\right)^{\left(1 / \gamma_{2}\right)}\right)^{\left(1 / \gamma_{1}\right)} .
$$

Summing the last inequality with the limit from $k_{3}$ to $\infty$, we obtain

$$
z\left(\overline{k_{3}}\right) \geq\left(M_{3} \beta\right) L^{1 / \gamma} \sum_{t_{2}=0}^{\infty} \frac{1}{a_{2}^{1 / \gamma_{1}}\left(\overline{k_{3}}+t_{2} \ell\right)}\left(\sum_{t_{1}=t_{2}}^{m_{2}(k)} \frac{1}{a_{1}^{1 / \gamma_{2}}\left(\overline{k_{2}}+t_{1} \ell\right)}\left(\left(\sum_{t=t_{1}}^{m_{1}(k)} \sum_{s=c}^{d} q\left(\overline{k_{1}}+t \ell, s\right)\right)\right)^{\left(1 / \gamma_{2}\right)}\right)^{\left(1 / \gamma_{1}\right)} .
$$

This contradicts to condition (11). Thus, $\beta=0$. Furthermore, the inequality $0<x(k)<z(k)$ implies that $\lim _{n \longrightarrow \infty} x(n)=0$. Now, the proof is complete.

Lemma 5. Let $x(k)$ be a positive solution of $(1)$, and $z(k)$ has the property $\left(P_{1}\right)$. Then,

$\left[a_{1}(k+\ell)\left[\Delta_{\ell}\left(a_{2}(k+\ell)\left[\Delta_{\ell} z(k+\ell)\right]^{\gamma_{1}}\right)\right]^{\gamma_{2}}\right]^{1 / \gamma} R(k) \leq \Delta_{\ell} z(k)$, from the Definition 1 and for all $k_{1} \geq k_{0}$, we have where $R(k)=\left(1 / a_{2}^{1 / \gamma_{1}}(k)\right)\left(\sum_{r=0}^{m_{1}(k)}\left(1 /\left(a_{1}^{1 / \gamma_{2}}\left(k_{1}+r \ell\right)\right)\right)\right)^{1 / \gamma_{1}}$.

Proof. Let $x(k)$ be a positive solution of (1). Since $z(k)$ has the property $\left(P_{1}\right)$, we know

$$
\Delta_{\ell}\left(a_{1}(k)\left[\Delta_{\ell}\left(a_{2}(k)\left[\Delta_{\ell} z(k)\right]^{\gamma_{1}}\right)\right]^{\gamma_{2}}\right)<0,
$$

$$
\begin{aligned}
a_{2}(k)\left[\Delta_{\ell} z(k)\right]^{\gamma_{1}} & =a_{2}\left(\overline{k_{1}}\right)\left[\Delta_{\ell} z\left(\overline{k_{1}}\right)\right]^{\gamma_{1}}+\sum_{r=0}^{m_{1}(k)} \Delta_{\ell}\left(a_{2}\left(\overline{k_{1}}+r \ell\right)\left[\Delta_{\ell} z\left(\overline{k_{1}}+r \ell\right)\right]^{\gamma_{1}}\right) \\
& \geq \sum_{r=0}^{m_{1}(k)} \frac{\left[a_{1}\left(\overline{k_{1}}+r \ell\right)\left[\Delta_{\ell}\left(a_{2}\left(\overline{k_{1}}+r \ell\right)\left[\Delta_{\ell} z\left(\overline{k_{1}}+r \ell\right)\right]^{\gamma_{1}}\right)\right]^{\gamma_{2}}\right]^{1 / \gamma_{2}}}{a_{1}^{1 / \gamma_{2}}\left(\overline{k_{1}}+r \ell\right)} \\
& \geq\left[a_{1}(k+\ell)\left[\Delta_{\ell}\left(a_{2}(k+\ell)\left[\Delta_{\ell} z(k+\ell)\right]^{\gamma_{1}}\right)\right]^{\gamma_{2}}\right]^{1 / \gamma_{2}} \sum_{r=0}^{m_{1}(k)} \frac{1}{a_{1}^{1 / \gamma_{2}}\left(\overline{k_{1}}+r \ell\right)} .
\end{aligned}
$$

Hence, we obtain

$$
\Delta_{\ell} z(k) \geq\left[a_{1}(k+\ell)\left[\Delta_{\ell}\left(a_{2}(k+\ell)\left[\Delta_{\ell} z(k+\ell)\right]^{\gamma_{1}}\right)\right]^{\gamma_{2}}\right]^{1 / \gamma} R(k) .
$$

This completes the proof.

\section{Main Results}

This section establishes criteria on oscillation and convergent solutions to (1) with the help of generalized Riccati transformation and Philos-type technique. Let us define functions $h, H: \mathbb{N}_{\ell} \times \mathbb{N}_{\ell} \longrightarrow \mathbb{R}$ such that 
(1) $H(k, k)=0$ with $k \geq k_{0} \geq 0$.

(2) $H(k, s)>0$ with $k>s \geq k_{0}$.

(3) $\Delta_{\ell(s)} H(k, s)=H(k, s+\ell)-H(k, s) \leq 0 \quad$ for $k>s \geq k_{0}$, and there exists a positive real sequence $\{\rho(k)\}$ such that

$$
\Delta_{\ell(s)} H(k, s)+\frac{\Delta_{\ell} \rho(s)}{\rho(s+\ell)} H(k, s)=-h(k, s) \sqrt{H(k, s)} .
$$

Theorem 1. Assume that $\{z(k)\}$ holds and there exists $\{\rho(k)\}$ a positive real-valued sequence in such a way that

$$
\lim _{k \rightarrow \infty} \sum_{r=0}^{m_{1}(k)}\left(C\left(\overline{k_{1}}+r \ell\right)-\frac{\gamma^{\gamma} B^{1+\gamma}\left(\overline{k_{1}}+r \ell\right)}{(1+\gamma)^{1+\gamma} A^{\gamma}\left(\overline{k_{1}}+r \ell\right)}\right)=\infty,
$$

where

$$
\begin{aligned}
& A(k)=\frac{\gamma \rho(k) R(k)}{\rho^{((\gamma+1) / \gamma)}(k+\ell)}, \\
& B(k)=\frac{\Delta_{\ell} \rho(k)}{\rho(k+\ell)}, \\
& C(k)=\rho(k) q_{1}(k), \\
& q_{1}(k)=L(1-p)^{\gamma} \sum_{s=c}^{d} q(k, s),
\end{aligned}
$$

and then, each solution of equation (1) is either $x(k) \longrightarrow 0$ as $k \longrightarrow \infty$ or oscillatory.

Proof. Suppose that $\{x(k)\}$ is a nonoscillatory solution of equation (1). By assuming $x(k)>0$ and $x(k+s \ell-\tau \ell)>0$ for $k \geq k_{1} \geq k_{0} \in \mathbb{N}_{\ell}$ and $\{z(n)\}$ satisfies two properties of Lemma 3 , we have

$$
x(k) \geq z(k)-\sum_{s=a}^{b} p(k, s) z(k+s \ell-\tau \ell) \geq\left(1-\sum_{s=a}^{b} p(k, s)\right) z(k) \geq(1-p) z(k)
$$

Using condition $\left(c_{3}\right)$ in equation $(1)$, we obtain

$$
\Delta_{\ell}\left(a_{1}(k)\left[\Delta_{\ell}\left(a_{2}(k)\left[\Delta_{\ell} z(k)\right]^{\gamma_{1}}\right)\right]^{\gamma_{2}}\right) \leq-\sum_{s=c}^{d} q(k, s) L x^{\gamma}(k+s \ell-\sigma \ell) .
$$

By applying equation (28) in the aforementioned inequality, we obtain

$$
\begin{aligned}
\Delta_{\ell}\left(a_{1}(k)\left[\Delta_{\ell}\left(a_{2}(k)\left[\Delta_{\ell} z(k)\right]^{\gamma_{1}}\right)\right]^{\gamma_{2}}\right) & \leq-L(1-p)^{\gamma} \sum_{s=c}^{d} q(k, s) z^{\gamma}(k+s \ell-\sigma \ell) \\
& \leq-q_{1}(k) z^{\gamma}(k+c \ell-\sigma \ell) .
\end{aligned}
$$

Define

$w(k)=\rho(k) \frac{a_{1}(k)\left[\Delta_{\ell}\left(a_{2}(k)\left[\Delta_{\ell} z(k)\right]^{\gamma_{1}}\right)\right]^{\gamma_{2}}}{z^{\gamma}(k)}, \quad k \geq k_{1}$.
Then, $w(k)>0$, for every $k \geq k_{1}$, and from equations (20) and (30), we have

$$
\Delta_{\ell} w(k) \leq-\rho(k) q_{1}(k)+\frac{\Delta_{\ell} \rho(k)}{\rho(k+\ell)} w(k+\ell)-\frac{\gamma \rho(k) R(k)}{\rho^{((\gamma+1) / \gamma)}(k+\ell)} w^{((\gamma+1) / \gamma)}(k+\ell) .
$$

The above equation is also expressed as 


$$
\Delta_{\ell} w(k) \leq-C(k)+B(k) w(k+\ell)-A(k) w^{((\gamma+1) / \gamma)}(k+\ell),
$$

where

$$
\begin{aligned}
& A(k)=\frac{\gamma \rho(k) R(k)}{\rho^{((\gamma+1) / \gamma)}(k+\ell)}, \\
& B(k)=\frac{\Delta_{\ell} \rho(k)}{\rho(k+\ell)}, \\
& C(k)=\rho(k) q_{1}(k) .
\end{aligned}
$$

Using the inequality,

$$
A u-B u^{((1+\beta) / \beta)} \leq \frac{\beta^{\beta}}{(1+\beta)^{1+\beta}} \times \frac{A^{1+\beta}}{B^{\beta}} .
$$

Now, using the above inequality, it is possible to write equation (33) as

$$
C(k)-\frac{\gamma^{\gamma}}{(1+\gamma)^{1+\gamma}} \times \frac{B^{1+\gamma}}{A^{\gamma}} \leq-\Delta_{\ell} w(k)
$$

By applying summation in the last inequality with the limits from $k_{1}$ to $k-\ell$, we have

$$
\sum_{r=0}^{m_{1}(k)}\left(C\left(\overline{k_{1}}+r \ell\right)-\frac{\gamma^{\gamma} B^{1+\gamma}\left(\overline{k_{1}}+r \ell\right)}{(1+\gamma)^{1+\gamma} A^{\gamma}\left(\overline{k_{1}}+r \ell\right)}\right) \leq w\left(\overline{k_{1}}\right)-w(k) \leq w\left(\overline{k_{1}}\right)
$$

from $w(k)>0$, which contradicts $(25)$ as $k \longrightarrow \infty$, and then, the solution $x(k)$ of $(1)$ is oscillatory. When $z(k)$ has property $\left(P_{2}\right)$, from $(11)$, we know $\lim _{k \longrightarrow \infty} x(k)=0$ by Lemma 4. The proof is complete.
Theorem 2. Suppose that (1) holds. If there exists $\{\rho(k)\}$ a positive real sequence such that

$$
\limsup _{k \rightarrow \infty} \frac{1}{H\left(k, k_{2}\right)} \sum_{r=0}^{m_{2}(k)}\left[H\left(k, \overline{k_{2}}+r \ell\right) C\left(\overline{k_{2}}+r \ell\right)-\frac{\gamma^{\gamma}}{(1+\gamma)^{1+\gamma}} \frac{\left[-h\left(k, \overline{k_{2}}+r \ell\right)\right]^{1+\gamma}}{A^{\gamma}\left(\overline{k_{2}}+r \ell\right) H^{((\gamma-1) / 2)}\left(k, \overline{k_{2}}+r \ell\right)}\right]=\infty .
$$

Subsequently, all solutions of equation (1) is either $x(k) \longrightarrow 0$ as $k \longrightarrow \infty$ or oscillatory.

Proof. Suppose that $\{x(k)\}$ is a nonoscillatory solution of equation (1). Proceeding as the proof of Theorem 1, we got equation (33). Now, multiplying inequality (33) by $H(k, s)$ and then summing the aforementioned inequality from $k_{2}$ to $k-\ell$, for all $k \geq k_{2} \geq k_{0}$, we have

$$
\begin{aligned}
\sum_{r=0}^{m_{2}(k)} H\left(k, \overline{k_{2}}+r \ell\right) C\left(\overline{k_{2}}+r \ell\right) \leq & -\sum_{r=0}^{m_{2}(k)} H\left(k, \overline{k_{2}}+r \ell\right) \Delta_{\ell} w\left(\overline{k_{2}}+r \ell\right) \\
& +\sum_{r=0}^{m_{2}(k)}\left(B\left(\overline{k_{2}}+r \ell\right) w\left(\overline{k_{2}}+r \ell+\ell\right)-A\left(\overline{k_{2}}+r \ell\right) w^{((\gamma+1) / \gamma)}\left(\overline{k_{2}}+r \ell+\ell\right)\right) H\left(k, \overline{k_{2}}+r \ell\right) .
\end{aligned}
$$

By summation by parts, we obtain

$$
\begin{aligned}
\sum_{r=0}^{m_{2}(k)} H\left(k, \overline{k_{2}}+r \ell\right) C\left(\overline{k_{2}}+r \ell\right) \leq & H\left(k, \overline{k_{2}}\right) w\left(\overline{k_{2}}\right) \\
& +\sum_{r=0}^{m_{2}(k)}\left[\Delta_{\ell\left(\overline{k_{2}}\right)} H\left(k, \overline{k_{2}}\right)+B\left(\overline{k_{2}}+r \ell\right) H\left(k, \overline{k_{2}}+r \ell\right)\right] w\left(\overline{k_{2}}+r \ell+\ell\right) \\
& -\sum_{r=0}^{m_{2}(k)} A\left(\overline{k_{2}}+r \ell\right) H\left(k, \overline{k_{2}}+r \ell\right) w^{((\gamma+1) / \gamma)}\left(\overline{k_{2}}+r \ell+\ell\right) .
\end{aligned}
$$


Using inequality (35), we have

$$
\begin{aligned}
\sum_{r=0}^{m_{2}(k)} H\left(k, \overline{k_{2}}+r \ell\right) C\left(\overline{k_{2}}+r \ell\right) \leq & H\left(k, \overline{k_{2}}\right) w\left(\overline{k_{2}}\right) \\
& +\sum_{r=0}^{m_{2}(k)} \frac{\gamma^{\gamma}}{(1+\gamma)^{1+\gamma}} \frac{\left[\Delta_{\ell\left(\overline{k_{2}}\right)} H\left(k, \overline{k_{2}}\right)+B\left(\overline{k_{2}}+r \ell\right) H\left(k, \overline{k_{2}}+r \ell\right)\right]^{1+\gamma}}{\left[A\left(\overline{k_{2}}+r \ell\right) H\left(k, \overline{k_{2}}+r \ell\right)\right]^{\gamma}} \\
& \cdot \sum_{r=0}^{m_{2}(k)}\left[H\left(k, \overline{k_{2}}+r \ell\right) C\left(\overline{k_{2}}+r \ell\right)-\frac{\gamma^{\gamma}}{(1+\gamma)^{1+\gamma}} \frac{\left[-h\left(k, \overline{k_{2}}+r \ell\right)\right]^{1+\gamma}}{A^{\gamma}\left(\overline{k_{2}}+r \ell\right) H^{((\gamma-1) / 2)}\left(k, \overline{k_{2}}+r \ell\right)}\right] \leq H\left(k, \overline{k_{2}}\right) w\left(\overline{k_{2}}\right),
\end{aligned}
$$

where

$$
\begin{aligned}
\Delta_{\ell\left(\overline{k_{2}}\right)} H\left(k, \overline{k_{2}}\right)+\frac{\Delta_{\ell} \rho\left(\overline{k_{2}}+r \ell\right)}{\rho\left(\overline{k_{2}}+r \ell+\ell\right)} H\left(k, \overline{k_{2}}+r \ell\right)= & -h\left(k, \overline{k_{2}}+r \ell\right) \sqrt{H\left(k, \overline{k_{2}}+r \ell\right)}, \\
& \cdot \frac{1}{H\left(k, \overline{k_{2}}\right)} \sum_{r=0}^{m_{2}(k)}\left[H\left(k, \overline{k_{2}}+r \ell\right) C\left(\overline{k_{2}}+r \ell\right)\right. \\
& \left.-\frac{\gamma^{\gamma}}{(1+\gamma)^{1+\gamma}} \frac{\left[-h\left(k, \overline{k_{2}}+r \ell\right)\right]^{1+\gamma}}{A^{\gamma}\left(\overline{k_{2}}+r \ell\right) H^{((\gamma-1) / 2)}\left(k, \overline{k_{2}}+r \ell\right)}\right] \leq w\left(\overline{k_{2}}\right),
\end{aligned}
$$

which is a contradiction to inequality (29). If $z(k)$ has satisfied the property (ii) of Lemma 3, then, by condition $(11)$, we have $x(k) \longrightarrow 0$ as $k \longrightarrow 0$. Hence, the theorem is proved.
Corollary 1. If $H(k, s)=(k-s)_{\ell}^{(m)}$ for all $k \geq s \geq 0$, $\rho(k)=1$, and

$$
\limsup _{k \rightarrow \infty} \frac{1}{k_{\ell}^{(m)}} \sum_{r=0}^{((k-s-\ell) / \ell)}\left[(k-\bar{s}-r \ell)_{\ell}^{(m)} q_{1}(\bar{s}+r \ell)-\frac{(1+\gamma)^{-(1+\gamma)}\left[-m \ell(k-\bar{s}-r \ell)_{\ell}^{((m / 2)-1)}\right]^{1+\gamma}}{R^{\gamma}(\bar{s}+r \ell)\left[(k-\bar{s}-r \ell)_{\ell}^{(m)}\right]^{((\gamma-1) / 2)}}\right]=\infty,
$$

for every $m \geq 1$, then each and every solution of equation (1) is oscillatory.
Corollary 2. If $H(k, s)=(\log ((k+\ell) /(s+\ell)))^{m}$, for all $k \geq s \geq 0, \rho(k)=1$, and

$$
\limsup _{k \rightarrow \infty} \frac{1}{(\log ((k+\ell) / \ell))^{m}} \sum_{r=0}^{((k-s-\ell) / \ell)}\left[\left(\log \frac{k+\ell}{\bar{s}+r \ell+\ell}\right)^{m} q_{1}(\bar{s}+r \ell)-\frac{(1+\gamma)^{-(1+\gamma)}(\log ((k+\ell) /(\bar{s}+r \ell+\ell)))^{(m-2)(1+\gamma)}}{R^{\gamma}(\bar{s}+r \ell)(\log ((k+\ell) /(\bar{s}+r \ell+\ell)))^{((m(\gamma-1)) / 2)}}\right]=\infty
$$


for every $m \geq 1$, then each and every solution of equation (1) is oscillatory.

Theorem 3. Suppose that condition (11) holds. Also, let

$$
0<\inf _{s \geq k_{0}}\left[\liminf _{k \rightarrow \infty} \frac{H(k, s)}{H\left(k, k_{0}\right)}\right] \leq \infty
$$

$$
\limsup _{k \rightarrow \infty} \frac{1}{H\left(k, k_{0}\right)} \sum_{r=0}^{m_{2}(k)} \frac{h^{2}\left(k, \overline{k_{2}}+r \ell\right)}{A\left(\overline{k_{2}}+r \ell\right) w^{((1-\gamma) / \gamma)}\left(\overline{k_{2}}+r \ell\right)}<\infty
$$

holds. If there is a sequence $\{\Phi(n)\}$ such that

$$
\sum_{r=0}^{m_{2}(k)} A\left(\overline{k_{2}}+r \ell\right) \Phi_{+}^{2}\left(\overline{k_{2}}+r \ell+\ell\right)=\infty
$$

$$
\limsup _{k \longrightarrow \infty} \frac{1}{H\left(k, k_{2}\right)} \sum_{r=0}^{m_{2}(k)}\left[H\left(k, \overline{k_{2}}+r \ell\right) C\left(\overline{k_{2}}+r \ell\right)-\frac{\left[h^{2}\left(k, \overline{k_{2}}+r \ell\right)\right]^{1+\gamma}}{4 A\left(k_{2}+r \ell\right) w^{((1-\gamma) / \gamma)}\left(k, \overline{k_{2}}+r \ell+\ell\right)}\right] \geq \Phi(s),
$$

where

$$
\Phi_{+}\left(k_{2}+r \ell+\ell\right)=\max \left\{\Phi\left(k_{2}+r \ell+\ell\right), 0\right\},
$$

where $A(k), B(k), C(k)$, and $h(k, s)$ are defined in (26) and (24), respectively, then each and every solution of equation (1) is either oscillatory or converges to zero.

Proof. Let $x(k)$ be a nonoscillatory solution of (1), and proceeding as in the proof of Theorem 2 , when $z(k)$ has a property $\left(P_{1}\right)$, from $(40)$, and rearranging, we obtain

$$
\begin{aligned}
w\left(\overline{k_{2}}\right) \geq & \limsup _{k \rightarrow \infty} \frac{1}{H\left(k, \overline{k_{2}}\right)} \sum_{r=0}^{m_{2}(k)}\left[H\left(k, \overline{k_{2}}+r \ell\right) C\left(\overline{k_{2}}+r \ell\right)-\frac{\left[h^{2}\left(k, \overline{k_{2}}+r \ell\right)\right]^{1+\gamma}}{4 A\left(k_{2}+r \ell\right) w^{((1-\gamma) / \gamma)}\left(k, \overline{k_{2}}+r \ell+\ell\right)}\right] \\
& +\liminf _{k \rightarrow \infty} \frac{1}{H\left(k, \overline{k_{2}}\right)} \sum_{r=0}^{m_{2}(k)}\left[\sqrt{H\left(k, \overline{k_{2}}+r \ell\right) A\left(\overline{k_{2}}+r \ell\right) w^{((\gamma+1) / \gamma)}\left(\overline{k_{2}}+r \ell+\ell\right)}\right. \\
& \left.+\frac{h\left(k, \overline{k_{2}}+r \ell\right)}{2 \sqrt{A\left(\overline{k_{2}}+r \ell\right) w^{((1-\gamma) / \gamma)}\left(k, \overline{k_{2}}+r \ell+\ell\right)}}\right]^{2},
\end{aligned}
$$

for $k \geq k_{2}$. It is derived from (48) that

$$
\begin{aligned}
w\left(\overline{k_{2}}\right) \geq & \Phi\left(\overline{k_{2}}\right)+\liminf _{k \rightarrow \infty} \frac{1}{H\left(k, \overline{k_{2}}\right)} \sum_{r=0}^{m_{2}(k)}\left[\sqrt{H\left(k, \overline{k_{2}}+r \ell\right) A\left(\overline{k_{2}}+r \ell\right) w^{((\gamma+1) / \gamma)}\left(\overline{k_{2}}+r \ell+\ell\right)}\right. \\
& \left.+\frac{h\left(k, \overline{k_{2}}+r \ell\right)}{\sqrt{A\left(\overline{k_{2}}+r \ell\right) w^{((1-\gamma) / \gamma)}\left(k, \overline{k_{2}}+r \ell+\ell\right)}}\right]^{2},
\end{aligned}
$$


which means that

$$
w\left(\overline{k_{2}}\right) \geq \Phi\left(\overline{k_{2}}\right),
$$

$$
\begin{aligned}
\liminf _{k \rightarrow \infty} \frac{1}{H\left(k, \overline{k_{2}}\right)} \sum_{r=0}^{m_{2}(k)}[ & \sqrt{H\left(k, \overline{k_{2}}+r \ell\right) A\left(\overline{k_{2}}+r \ell\right) w^{((\gamma+1) / \gamma)}\left(\overline{k_{2}}+r \ell+\ell\right)} \\
& \left.+\frac{h\left(k, \overline{k_{2}}+r \ell\right)}{\sqrt{A\left(\overline{k_{2}}+r \ell\right) w^{((1-\gamma) / \gamma)}\left(k, \overline{k_{2}}+r \ell+\ell\right)}}\right]^{2}<\infty .
\end{aligned}
$$

Therefore,

$$
\begin{aligned}
\liminf _{k \rightarrow \infty}\left[\frac{1}{H\left(k, \overline{k_{2}}\right)} \sum_{r=0}^{m_{2}(k)} H\left(k, \overline{k_{2}}+r \ell\right) A\left(\overline{k_{2}}+r \ell\right) w^{((\gamma+1) / \gamma)}\left(\overline{k_{2}}+r \ell+\ell\right)\right. \\
+\frac{1}{H\left(k, \overline{k_{2}}\right)} \sum_{r=0}^{m_{2}(k)} h\left(k, \overline{k_{2}}+r \ell\right) \sqrt{H\left(k, \overline{k_{2}}+r \ell\right)} w\left(\overline{k_{2}}+r \ell+\ell\right) \\
\left.+\frac{1}{4 H\left(k, \overline{k_{2}}\right)} \sum_{r=0}^{m_{2}(k)} \frac{h^{2}\left(k, \overline{k_{2}}+r \ell\right)}{A\left(\overline{k_{2}}+r \ell\right) w^{((1-\gamma) / \gamma)}\left(\overline{k_{2}}+r \ell+\ell\right)}\right]<\infty .
\end{aligned}
$$

Then,

$$
\begin{gathered}
\liminf _{k \rightarrow \infty}\left[\frac{1}{H\left(k, \overline{k_{2}}\right)} \sum_{r=0}^{m_{2}(k)} H\left(k, \overline{k_{2}}+r \ell\right) A\left(\overline{k_{2}}+r \ell\right) w^{((\gamma+1) / \gamma)}\left(\overline{k_{2}}+r \ell+\ell\right)\right. \\
\left.+\frac{1}{H\left(k, \overline{k_{2}}\right)} \sum_{r=0}^{m_{2}(k)} h\left(k, \overline{k_{2}}+r \ell\right) \sqrt{H\left(k, \overline{k_{2}}+r \ell\right)} w\left(\overline{k_{2}}+r \ell+\ell\right)\right]<\infty .
\end{gathered}
$$

The abovementioned inequality can also be expressed as

$$
\liminf _{k \rightarrow \infty}[U(k)+V(k)]<\infty, \quad \text { for } k \geq k_{2},
$$

$$
\begin{aligned}
& U(k)=\frac{1}{H\left(k, \overline{k_{2}}\right)} \sum_{r=0}^{m_{2}(k)} H\left(k, \overline{k_{2}}+r \ell\right) A\left(\overline{k_{2}}+r \ell\right) w^{((\gamma+1) / \gamma)}\left(\overline{k_{2}}+r \ell+\ell\right) \\
& V(k)=\frac{1}{H\left(k, \overline{k_{2}}\right)} \sum_{r=0}^{m_{2}(k)} h\left(k, \overline{k_{2}}+r \ell\right) \sqrt{H\left(k, \overline{k_{2}}+r \ell\right)} w\left(\overline{k_{2}}+r \ell+\ell\right) .
\end{aligned}
$$

Here, we assert

$$
\sum_{r=0}^{m_{2}(k)} A\left(\overline{k_{2}}+r \ell\right) w^{((\gamma+1) / \gamma)}\left(\overline{k_{2}}+r \ell+\ell\right)<\infty
$$

Conversely, suppose that

$$
\sum_{r=0}^{m_{2}(k)} A\left(\overline{k_{2}}+r \ell\right) w^{((\gamma+1) / \gamma)}\left(\overline{k_{2}}+r \ell+\ell\right)=\infty .
$$

From equation (45), we have

$$
\inf _{s \geq k_{0}}\left[\liminf _{k \longrightarrow \infty} \frac{H(k, s)}{H\left(k, k_{0}\right)}\right]>\mu,
$$

for $\mu>0$; then, $\left(H(k, s) / H\left(k, k_{0}\right)\right)>\mu$ for $k \geq k_{2} \geq k_{1}$. There exists a positive constant $M_{4}>0$ such that

$$
\sum_{r=0}^{m_{2}(k)} A\left(\overline{k_{2}}+r \ell\right) w^{((\gamma+1) / \gamma)}\left(\overline{k_{2}}+r \ell+\ell\right) \geq \frac{M_{4}}{\mu} .
$$

Thus, for $k \geq k_{3}$ and using equation (74), we have 


$$
\begin{aligned}
U(k)= & \frac{1}{H\left(k, \overline{k_{2}}\right)} \sum_{r=0}^{m_{2}(k)} H\left(k, \overline{k_{2}}+r \ell\right) \Delta_{\ell}\left(\sum_{r_{1}=0}^{\left(\left(r-\overline{k_{2}}-\ell\right) / \ell\right)} A\left(\overline{k_{2}}+r_{1} \ell\right) w^{((\gamma+1) / \gamma)}\left(\overline{k_{2}}+r_{1} \ell+\ell\right)\right) \\
& +A\left(\overline{k_{2}}+r \ell\right) w^{((\gamma+1) / \gamma)}\left(\overline{k_{2}}+r \ell+\ell\right) \\
= & -\frac{1}{H\left(k, \overline{k_{2}}\right)} \sum_{r=0}^{\left(\left(k-\overline{k_{3}}-\ell\right) / \ell\right)}\left(\sum_{r_{1}=0}^{\left(\left(r-\overline{k_{2}}-\ell\right) / \ell\right)} A\left(\overline{k_{2}}+r_{1} \ell\right) w^{((\gamma+1) / \gamma)}\left(\overline{k_{2}}+r_{1} \ell+\ell\right)\right) \Delta_{\ell\left(\overline{k_{2}}\right)} H\left(k, \overline{k_{3}}+r \ell\right) \\
& -\frac{A\left(\overline{k_{2}}\right) w^{((\gamma+1) / \gamma)}\left(\overline{k_{2}}+\ell\right)}{H\left(k, \overline{k_{2}}\right)} \sum_{r=0}^{\left(\left(k-\overline{k_{3}}-\ell\right) / \ell\right)} H\left(k, \overline{k_{3}}+r \ell\right) A\left(\overline{k_{2}}+r \ell\right) w^{((\gamma+1) / \gamma)}\left(\overline{k_{2}}+r \ell+\ell\right) \\
\geq & \frac{1}{H\left(k, \overline{k_{2}}\right)} \sum_{r=0}^{\left(\left(k-\overline{k_{3}}-\ell\right) / \ell\right)}\left(\sum_{r_{1}=0}^{\left(\left(r-\overline{k_{2}}-\ell\right) / \ell\right)} A\left(\overline{k_{2}}+r_{1} \ell\right) w^{((\gamma+1) / \gamma)}\left(\overline{k_{2}}+r_{1} \ell+\ell\right)\right)\left(-\Delta_{\ell\left(\overline{k_{2}}\right)} H\left(k, \overline{k_{3}}+r \ell\right)\right) \\
\geq & \frac{M_{4} H\left(k, \overline{k_{2}}\right)}{\mu H\left(k, \overline{k_{3}}\right)} \geq \sum_{r=0} \geq M_{4} .
\end{aligned}
$$

Since $M_{4}$ is an arbitrary constant,

$$
\lim _{n \longrightarrow \infty} V\left(k_{n}\right)=-\infty .
$$

$$
\lim _{k \longrightarrow \infty} U(k)=\infty .
$$

Furthermore, consider a sequence $k_{n} \longrightarrow \infty$ such that

$\lim _{n \longrightarrow \infty}\left[U\left(k_{n}\right)+V\left(k_{n}\right)\right]=\liminf _{k \longrightarrow \infty}[U(k)+V(k)]$.

From (56), there exists a number $M_{5}$ such that

$$
U\left(k_{n}\right)+V\left(k_{n}\right) \leq M_{5}, \quad \text { for } n=0,1,2, .
$$

Resulting from (64), we conclude that
By (65), for a large value of $n$, we have

$$
1+\frac{V\left(k_{n}\right)}{U\left(k_{n}\right)} \leq \frac{M_{5}}{U\left(k_{n}\right)}<\frac{1}{2} .
$$

From (66), we obtain

$$
\lim _{n \longrightarrow \infty} \frac{V^{2}\left(k_{n}\right)}{U\left(k_{n}\right)}=\infty .
$$

Nevertheless, by Schwarz's inequality, we have

$$
\begin{aligned}
V^{2}\left(k_{n}\right)= & \left(\frac{1}{H\left(k_{n}, \overline{k_{2}}\right)} \sum_{r=0}^{\left(\left(k_{n}-\overline{k_{2}}-\ell\right) / \ell\right)} h\left(k_{n}, \overline{k_{2}}+r \ell\right) \sqrt{H\left(k_{n}, \overline{k_{2}}+r \ell\right)} w\left(\overline{k_{2}}+r \ell+\ell\right)\right)^{2} \\
\leq & \left(\frac{1}{H\left(k_{n}, \overline{k_{2}}\right)} \sum_{r=0}^{\left(\left(k_{n}-\overline{k_{2}}-\ell\right) / \ell\right)} H\left(k_{n}, \overline{k_{2}}+r \ell\right) A\left(\overline{k_{2}}+r \ell\right) w^{((\gamma+1) / \gamma)}\left(\overline{k_{2}}+r \ell+\ell\right)\right) \\
& \cdot\left(\frac{1}{H\left(k_{n}, \overline{k_{2}}\right)} \sum_{r=0}^{\left(\left(k_{n}-\overline{k_{2}}-\ell\right) / \ell\right)} \frac{h^{2}\left(k_{n}, \overline{k_{2}}+r \ell\right)}{A\left(\overline{k_{2}}+r \ell\right) w^{((1-\gamma) / \gamma)}\left(\overline{k_{2}}+r \ell+\ell\right)}\right) \\
\leq & U\left(k_{n}\right)\left(\frac{1}{H\left(k_{n}, \overline{k_{2}}\right)} \sum_{r=0}^{\left(\left(k_{n}-\overline{k_{2}}-\ell\right) / \ell\right)} \frac{h^{2}\left(k_{n}, \overline{k_{2}}+r \ell\right)}{A\left(\overline{k_{2}}+r \ell\right) w^{((1-\gamma) / \gamma)}\left(\overline{k_{2}}+r \ell+\ell\right)}\right) .
\end{aligned}
$$


Consequently,

$$
\begin{aligned}
\frac{V^{2}\left(k_{n}\right)}{U\left(k_{n}\right)} & \leq \frac{1}{H\left(k_{n}, \overline{k_{2}}\right)} \sum_{r=0}^{\left(\left(k_{n}-\overline{k_{2}}-\ell\right) / \ell\right)} \frac{h^{2}\left(k_{n}, \overline{k_{2}}+r \ell\right)}{A\left(\overline{k_{2}}+r \ell\right) w^{((1-\gamma) / \gamma)}\left(\overline{k_{2}}+r \ell+\ell\right)} \\
& \leq \frac{1}{\mu H\left(k_{n}, k_{0}\right)} \sum_{r=0}^{\left(\left(k_{n}-\overline{k_{2}}-\ell\right) / \ell\right)} \frac{h^{2}\left(k_{n}, \overline{k_{2}}+r \ell\right)}{A\left(\overline{k_{2}}+r \ell\right) w^{((1-\gamma) / \gamma)}\left(\overline{k_{2}}+r \ell+\ell\right)} .
\end{aligned}
$$

It follows from (68) that

$$
\lim _{n \longrightarrow \infty} \frac{1}{H\left(k_{n}, k_{0}\right)} \sum_{r=0}^{\left(\left(k_{n}-k_{2}-\ell\right) / \ell\right)} \frac{h^{2}\left(k_{n}, k_{2}+r \ell\right)}{A\left(k_{2}+r \ell\right) w^{((1-\gamma) / \gamma)}\left(\overline{k_{2}}+r \ell+\ell\right)}=\infty
$$

which is a contradiction to inequality (46). Hence, by (52), equation (72) holds:

$$
\sum_{r=0}^{m_{2}(k)} A\left(\overline{k_{2}}+r \ell\right) \Phi_{+}^{2}\left(\overline{k_{2}}+r \ell+\ell\right) \leq \sum_{r=0}^{m_{2}(k)} A\left(\overline{k_{2}}+r \ell\right) w^{2}\left(\overline{k_{2}}+r \ell+\ell\right)<\infty
$$

which is a contradiction to inequality (47) and concludes the proof. If $z(k)$ fulfills the property $\left(P_{2}\right)$ of Lemma 3 , then, by $\liminf _{k \rightarrow \infty} \frac{1}{H\left(k, k_{0}\right)} \sum_{r=0}^{\left(\left(k-k_{2}-\ell\right) / \ell\right)} H(k, s+r \ell) C(s+r \ell)<\infty$ equation (11), we have obtained $x(k) \longrightarrow 0$ as $k \longrightarrow \infty$. Hence, the proof is complete.

Theorem 4. Suppose that all conditions of Theorem 3 are satisfied excluding condition (46). Also, let

$$
\liminf _{k \rightarrow \infty} \frac{1}{H(k, s)} \sum_{r=0}^{((k-s-\ell) / \ell)}\left[H(k, s+r \ell) C(s+r \ell)-\frac{h^{2}(k, s+r \ell)}{4 A(s+r \ell)}\right] \geq \Phi(s) .
$$

Furthermore, each and every solution of equation (1) is either $x(k) \longrightarrow 0$ as $k \longrightarrow \infty$ or oscillatory.

Proof. The proof is approximately the same as Theorem 3, and in consequence, the details are excluded.
Example 1. The third-order neutral generalized difference equation with distributed delay is given as

$$
\Delta_{\ell}\left(\Delta_{\ell}\left(\Delta_{\ell}\left(x(k)+\sum_{s=2}^{3} \frac{1}{s} x(k+s \ell-\ell)\right)\right)^{3}\right)^{(1 / 3)}+\sum_{s=1}^{3} \frac{2^{7 / 3}}{s} x(k+s \ell-\ell)=0
$$

Here, $\quad a_{1}(k)=a_{2}(k)=1, \quad \gamma_{1}=3, \quad \gamma_{2}=(1 / 3), \quad$ and $\quad \rho(k)=1 . \quad$ Then, $\quad R(k)=\left(\left(k-k_{1}\right) / \ell\right) \quad$ and $p(k, s)=(1 / s), \quad q(k, s)=2^{7 / 3}((k / 2)+(1 / s)), \quad \tau=1, \quad \sigma=1, \quad q_{1}(k)=\left(2^{1 / 3} L / 9\right)(6 k+11)$ which imply 


$$
\lim _{k \rightarrow \infty} \sum_{r=0}^{m_{1}(k)} \frac{2^{1 / 3} L}{9}\left(6 k_{1}+6 r \ell+11\right)=\infty .
$$

Henceforth, by Theorem 1, each and every solution of equation (75) is oscillatory. Moreover, $\{x(n)\}=\left\{(-1)^{[n / \ell]}\right\}$ is one such oscillatory solution of equation (75).

\section{Conclusion}

In this paper, we present the qualitative properties such as convergence and oscillatory behaviors of third-order nonlinear neutral distributed-delay generalized difference equation. The results we obtained in this paper for difference equation involving generalized difference operator $\Delta_{\ell}$ with distributed delay are rare and new in the literature. Also, the technique adopted is different from that which already exists. The significance of the results is also well established by an example presented in this paper.

\section{Data Availability}

No data were used to support this study.

\section{Conflicts of Interest}

The authors declare that they have no conflicts of interest.

\section{References}

[1] R. P. Agarwal, Difference Equations and Inequalities, Theory, Methods and Applications, Marcel Dekker, New York, NY, USA, 2nd edition, 2000.

[2] R. Saravanakumar, M. S. Ali, J. Cao, and H. Huang, "Ho state estimation of generalised neural networks with interval timevarying delays," International Journal of Systems Science, vol. 47, no. 16, pp. 3888-3899, 2016.

[3] M. Syed Ali, G. Narayanan, S. Sevgen, V. Shekher, and S. Arik, "Global stability analysis of fractional-order fuzzy BAM neural networks with time delay and impulsive effects," Communications in Nonlinear Science and Numerical Simulation, vol. 78, p. 104853, 2019.

[4] M. Syed Ali, P. Balasubramaniam, and Q. Zhu, "Stability of stochastic fuzzy BAM neural networks with discrete and distributed time-varying delays," International Journal of Machine Learning and Cybernetics, vol. 8, no. 1, pp. 263-273, 2017.

[5] M. S. Ali, "Stability analysis of Markovian jumping stochastic Cohen-Grossberg neural networks with discrete and distributed time varying delays," Chinese Physics B, vol. 23, no. 6, Article ID 060702, 2014.

[6] M. Syed Ali, N. Gunasekaran, and M. Esther Rani, "Robust stability of hopfield delayed neural networks via an augmented L-K functional," Neurocomputing, vol. 234, pp. 198204, 2017.

[7] R. P. Agarwal, M. Bohner, S. R. Grace, and D. O’Regan, Discrete Oscillation Theory, Hindawi Publishers, New York, NY, USA, 2005.

[8] S. R. Grace, J. R. Graef, and E. Tunç, "Oscillatory behaviour of third order nonlinear differential equations with a nonlinear nonpositive neutral term," Journal of Taibah University for Science, vol. 13, no. 1, pp. 704-710, 2019.
[9] M. F. Aktas, A. Tiryaki, and A. Zafer, "Oscillation of the thirdorder nonlinear delay difference equations," Turkish Journal of Mathematics, vol. 36, pp. 422-436, 2012.

[10] E. M. Elabbasy, M. Y. Barsom, and F. S. AL-dheleai, "New oscillation criteria for third-order nonlinear mixed neutral difference equations," Chinese Journal of Mathematics, vol. 2014, Article ID 676470, 11 pages, 2014.

[11] P. Dinakar, S. Selvarangam, and E. Thandapani, "Oscillation of nonlinear third order perturbed functional difference equations," Nonautonomous Dynamical Systems, vol. 6, no. 1, pp. 57-64, 2019.

[12] I. Gyori and G. Ladas, Oscillation Theory of Delay Differential Equations with Applications, Clarendon Press, Oxford, UK, 1991.

[13] D. Levi, S. Termblay, and P. Winterniz, "Lie point symmetries of difference equations and lattices," Journal of Physics A Mathematical and General, vol. 33, no. 47, pp. 8501-8523, 2001.

[14] D. Levi, S. Tremblay, and P. Winternitz, "Lie symmetries of multidimensional difference equations," Journal of Physics A: Mathematical and General, vol. 34, no. 44, pp. 9507-9524, 2001.

[15] D. Levi and P. Winterniz, "Continuous symmetries of difference equations," Journal of Physics A: Mathematical and General, vol. 39, no. 2, 2005.

[16] M. Maria Susai Manuel, G. Britto Antony Xavier, and E. Thandapani, "Theory of generalized difference operator and its applications," Far East Journal of Mathematical Science, vol. 20, no. 2, pp. 163-171, 2006.

[17] S. N. Elaydi, An Introduction to Difference Equations, Springer, Berlin, Germany, Third edition, 2000.

[18] E. Schmeidal, "Oscillatory and asymptotically zero soluions of third order difference equations with quasi differences," Opuscula Mathematica, vol. 26, no. 2, pp. 361-369, 2006.

[19] S. Saker, J. O. Alzabut, and A. Mukheimer, "On the oscillatory behavior for a certain class of third order nonlinear delay difference equations," Electronic Journal of Qualitative Theory of Differential Equations, vol. 67, no. 67, pp. 1-16, 2010.

[20] B. Selvaraj and I. M. A. Jaffer, "On the oscillation of the solution to third order nonlinear difference equations," The Journal of Mathematics and Computer Science, vol. 7, pp. 873-876, 2010.

[21] B. Selvaraj, P. Mohankumar, and A. Balasubramanian, "Oscillatory solutions of certain third order non-linear difference equations," International Journal of Nonlinear Science, vol. 14, pp. 216-219, 2012.

[22] B. Selvaraj, P. Mohankumar, and V. Ananthan, "Oscillatory and non oscillatory behavior of neutral delay difference equations," International Journal of Nonlinear Science, vol. 13, pp. 472-474, 2012.

[23] E. Thandapani, S. Selvarangam, and S. Devarajalu, "Oscillatory behavior of third order nonlinear difference equation with mixed neutral terms," Electronic Journal of Qualitative Theory of Differential Equations, vol. 53, no. 53, pp. 1-11, 2014. 